\title{
Pre-Antiretroviral Care and Antiretroviral Therapy for HIV/Aids
}

\author{
Hemalatha $\mathrm{S}^{1^{*}}$, Menaka $\mathrm{K}^{2}$, Kumutha $\mathrm{T}^{3}$, Manisha. $\mathrm{K}^{4}$ \\ ${ }^{1,2,3}$ Associate Professor Department of Pharmacy Practice, Nandha College of \\ Pharmacy, Erode, Tamilnadu \\ ${ }^{4}$ Assistant Professor Department of Obstetrics and Gynecological Nursing, Nandha \\ College of Nursing, Erode, Tamilnadu \\ Email: hema.mpharm@gmail.com¹, kmenaka1980@gmail.com², \\ Kumuthapharmacy@gmail.com³ ${ }^{3}$ Manishabsc2017@gmail.com ${ }^{4}$ \\ ${ }^{*}$ Corresponding Author
}

\begin{abstract}
In order to advise policies and key arrangements for improved systemic HIV/AIDS service, this paper addresses HIV follow-up take-up and hardship under pre-ARV treatment and deferred ART start. Satisfactory post-test advice for recently assessed PLHIV, coupled with follow-up consideration by specialists in network support, may help retain PLHIV in the pre-ARV brain and enable ART to be launched in a timely manner. In relation to prepared and directed conventional/otherworldly healers who could supplement government efforts in contribution, a few parts of pre-ARV care are being explored.
\end{abstract}

Keywords: ARV, HIV/AIDS, pre-ARV.

\section{Introduction}

The epidemic has destroyed the present economic turn of events and has ultimately limited the future of two or three nations. In addition, in several assetweak situations, the generally unreliable mechanisms of clinical logic have been over-expanded. As of now, HIV/AIDS reports on the summary of the world's top five suicide driving targets, and is responsible for one in every five passes. In any case, achieving this objective requires a persuasive and complete approach to HIV/AIDS that is ideally designed to ensure the far-reaching involvement of PLHIV and its alliances in HIV research, repudiation, diagnosis, care and financing. As such, the solution requires the need to scale up HIV testing to differentiate all those receiving HIV treatment and to strengthen the connection between HIV validation and complete HIV/AIDS care, including the ideal beginning of ART.

In any case, the general standard targets persistent incitement and specific pre-ARV treatment for currently screened PLHIV once usually (for WHO phases 1 and 2) on the off chance of no misunderstanding. In either scenario, with the cotrimoxazole prophylaxis scheme forestalling ambitious contaminations such as Pneumocystis carinii pneumonia and others, the rules recommend quarterly tests of PLHIV not yet eligible for ART provided no health complications arise and the prescriptions are feasibly consumed. Standard preARV treatment ensures that PLHIV is seen to initiate
ART on time, among others. Accessible affirmation indicates that traditional pre-ARV treatment increases the likelihood of beginning ART on time by several incidents, with a customary 15 years of life putting everything in a stable position for someone on long-term counseling. Low take-up or episode to follow-up of currently screened PLHIV under pre-ARV treatment and the beginning of ART are enormous difficulties in scaling up acceptance for full HIV care, particularly where efforts are generally guided before the birthplace of ART.

A sensibly modern miracle in the HIV/AIDS association is exhaustive HIV/AIDS treatment and backing. The definition establishes a detailed stepping stool of ascendance from HCT to ART pre-ARV treatment. It is equally planned for PLHIV with regard to those apparently affected by HIV (families, partners, youths and the individuals who give care and backing from the area). At all periods of HIV disease, the assistance endeavors to adapt to the detailed needs of PLHIV and attempts to delay the turn of events and preserve the person at the most simple even minded level. Its little spaces reinforce HCT and PLHIV's therapeutic, psychosocial, sound, actual, monetary, family social and community approach. The PLHIV itself or those impacted by HIV should be merged in the decision, strategy and creation of the partnerships with an acceptable concept and assistance for HIV. The fair approval of organizations, association details and consumer self-sufficiency should be equally assured. 


\section{HIV Counselling Test}

The main area for all HIV evasion, therapy, rehabilitation and support services is the HIV Counselling Exam. People learn their HIV status and its advice by HIV Counselling Assessments and make informed decisions about their future lives. The HIV Therapy Test decreases the risk of developing or transmitting HIV by means of healthy social reform. In addition, it stimulates exposure and social assistance and lowers denial, disgrace, and loneliness. Mediation with HIV-shirking started at the potential of thinking by the individual. This helps the customer, with the aid of a guide, to secretly investigate and receive his/her HIV hazards and to gain potential with his/her HIV defilement status. HIV Counseling Test ought to be conceivable in zones with no real plan.

It creates "pick in" in which each patient is regularly endorsed and given an HIV test and the patient expressly consents to accept the HIV test. In comparison, if an HIV test is regularly endorsed and issued to each patient, it can be "quit" and the patient is taught to minimize the test with regard to his/her right. In particular cases, for example, tissue blessing and lawful requirements for medication.

\section{Pre-Antiretroviral (Pre-ARV) Care}

PLHIV is given Pre-ARV treatment until they are prepared for Painting. It develops psychosocial maintenance, thinking empowerment, reward development, prophylaxis of cotrimoxazole to thwart cunning infections and routine PLHIV testing to determine their ART ability. Subsequently, several PLHIV lost to follow-up do not benefit from the general inclinations of prosperity that this thinking is intended to offer. Little barriers to the take-up of preARV treatment are considered, and the sociorelevant and methodological issues for pre-ARV care may relate extraordinarily to those for ART. For eg, pre-ARV customers are inclined to be very powerful and their work is often diverged from ART-qualified customers by circumventing. Therefore, if they do not take pre-ARV treatment, they do not believe themselves to be vulnerable against any threat, disregarding ART-qualified clients that are more likely to be cleaned out sufficiently to justify pursuit of clinical administration. A whole load of research has been carried out on challenges to sustaining and adhering to ART in both high and low pay environments (some of the limitations perceived by these tests consolidate energy age, low guidance status, money-related limits, poor structure of clinical administration, Small and insufficiently coordinated workers of prosperity, imminent downside, non-appearance of social aid, nonattendance of deterioration reveals of naughtiness and defenseless stock of drugs. Others improve comorbidities, deficiencies, hunger, keeping up time and travel prices, drug outcomes, inability to move on developmental drugs, depression, emotions, uncertainty of outcomes, exacting procedures and negligence. In any case, little clarity behind setback to follow-up of PLHIV under pre-ARV is contemplated, especially with regard to Uganda where HIV and ART treatment is set to be high.

\section{Antiretroviral Therapy (ART)}

Workmanship incorporates the use of three ARV medications at any time to maximally cover HIV and avoid HIV sickness growth. Immense reductions in mortality and suffering rates have been identified where an extraordinary ARV schedule is used. In lowand-focus pay countries, 5 million out of around 10 million individuals who needed ART got it by and wide. This also means that 8 million PLHIVs requiring ART could not get to ART in these nations. The new WHO rules from 2010 on the edge of starting ART would dramatically increase the amount of PLHIV that needs ART, especially in vulnerable resource settings. In addition, the latest proposal increases the conversation about whether PLHIVs launch ART at a CD4 cell count. Deferred initiation of ART for PLHIV is a vital issue, especially when WHO supports earlier ART starting with a CD4 cell count. Delayed initiation of ART is linked to late-stage findings and results in horror and mortality associated with HIV in high areas. Nonetheless, restricted recognition and delayed authorization of HIV organisations are up to now a crucial prevention of these objectives (UAC, 2007). In a country where HIV and ART treatment are likely to be strong, there is a lack of information about why PLHIV will start ART so late.

\section{Health Care Framework}

Many multilateral associations and humanitarian programs, for example, UNICEF/WHO have sponsored an integrated local area case solution for fevers in children to meet vulnerable people, in recognition of insecurity and a small well-being base for extending admission to the provincial poor and generally defenseless. Similarly, geographically situated HIV/ART consideration systems steered in multiple asset-helpless areas have also indicated increased take-up, strong HIV treatment consistency levels and expanded ART conformity. While these pilot considerations have been discussed in extraordinarily subsidized situations, their prosperity offers the impetus for a tentative intercession of limited initiative adapted to the optimal usage of available properties as long as they improve entry to farreaching HIV treatment. Local area well-being staff 18 or organisations help HIV care professionals, such as offering a network of trained and guided volunteers to broaden admission to far-reaching HIV services. HIV/AIDS is a disease characterized by guilt, discipline, isolation, and an outstanding social assistance obligation for families and local communities. In addition, the situation needs social reform for the PLHIV to enter administrations with counteraction, treatment and backing. Exploration has shown that the actions of persons are influenced by multiple factors operating at different stages, all 
in all, from the intrapersonal and emotional to the local area and community.

\section{Low-Cost HIV Care}

Due to dejection and a minimal establishment of prosperity for the expansion of permission to the common poor and broadly exposed, numerous multilateral affiliations and prosperity programs, such as UNICEF/WHO, have sponsored a collaborative neighborhood solution for fevers in children to occur in destitute persons. In comparison, HIV/ART privately organized thinking projects directed in diverse resource-impotent contexts have similarly demonstrated extended take-up, high levels of quality for HIV treatment and extended commitment to ART. Yet in exceedingly funded situations, these pilot factors have been orchestrated, their thriving gives the impetus to a primer of negligible exertion mediations redid to the ideal use of the services available, as long as they improve the induction to sweeping HIV treatment. For eg, community works 18 or organization sustain HIV care specialists have a pool of workable and directed volunteers for increasing induction of sweep HIV care. HIV/AIDS is a disease represented by disgrace, power, detachment and an extraordinary request for support from family and neighborhood. Furthermore, for the PLHIV to get to neutralization, treatment and sponsorship organisations, the situation requires social reform. Research has shown that the decisions of people are impacted by different influences that function at different stages, ranging from intrapersonal and psychological to neighborhood culture with all taken into account.

\section{Data Collection For ART}

Singular evaluation is a pattern of perception of solicitation based on undeniable methodological traditions of solicitation that explore a social or human issue. The specialist gathers a complex, detailed image, words of examination, reports pointby-point interviews with sources and coordinates the evaluation in a trademark environment. It helps the researcher to get a perception of their observations, concepts, emotions, characteristics, understanding and points of view against a thing, organization, thinking or different musings from a social event of individuals. In a natural social event freeing up where people are to chat with each other, requests are sent. The components of the social occasion energize the blend of decently unmistakable knowledge on inescapable mentalities, contemplations, disputes and irregularities. The interaction between the people analyzing the environment of the individuals centered points of view on the topic of research. Peculiar studies to investigate the theoretical implications and understandings that are tailored to individuals' interactions. And get data from people who are in a position to explain exactly why they do it, what people or the neighborhood do. The explanation that they have unique assets that make them capable of the topic of interest is usually defined by key observers. The people were intentionally scanned government workplaces as no non-authoritative focuses provided wide-ranging HIV treatment in the region during the time under review.

Quantitative systems are analysis tools used to gather quantifiable knowledge overseeing statistics and separate items of assessment. They are used to evaluate a hypothesis or to select a relation between factors that are enlightening (free) and resulting (subordinate). In order to present the delayed effects of these methodologies, projections, outlines, tables and graphs are also used. From a wider point of view, the articulation "mediation" implies any effort offered to inspect individuals that are likely to harm their position of success or the after-effect of concern. If randomization is conducted correctly, the individuals' benchmark features are most likely to be virtually similar in all social occasions. Due to the propensity of randomization assurance, all recognized and dark prognostic components in the medication errand are hereafter changed. In brief, 31 experiments in which experts at any rate learn two interventions in a movement of people who get them in discretionary solicitation are quantitative, near, regulated.

HIV/AIDS social order keeping experts are good neighborhood volunteers or ART ace clients who have been honed and selected to organize and inspire PLHIV to look for treatment and connect them with the specialist groups in this way. A provided exploration office master who completed the HIV testing was enrolled in each center section. The specialist at each center stated that the certified customer picks and opens an envelope from a lot of four for business to the fitting arm after a customer had tried HIV-positive. This framework persisted at each center until it was sorted out by the critical assigned numbers per weapon. It was impossible to protect the staff of the multiple weapons, considering the likelihood of intercession, as to which weapon they had a position in at the end of the day.

\section{Loss To Follow-Up Of HIV Under Pre-ARV Care}

Purposes behind leaving pre-ARV care that emerged out of this examination included inadequate directing, contention from standard/extraordinary healers, transportation costs, holding up time, nonattendance of inspirations and sex irregularity. Nonappearance of transport was every now and again referred to as a limit to standard pre-ARV interest. Some PLHIV experienced issues bearing the expense of transport costs to go to the units for the organizations aside from in the event that they got security maintain. Others got a kick out of the chance to use the insignificant consumption they expected to purchase the cotrimoxazole locally. Nonattendance of sparks was another reality for loss of follow up conveyed that neither the prosperity workers nor the respondents had totally understood the benefits of offering or going to pre-ARV care. Some prosperity 
workers might not want to zero in on PLHIV without clear results, given the high leftover main job and few resources they had. Sex uniqueness imparted the fear of being beaten or isolated by their mates as an inspiration driving why various women didn't go to the pre-ARV focus, regardless, when they had attempted and acknowledged they were HIV-positive.

\section{Delayed ART Initiation}

The late initiators much of the time declared that the lacking stock or outright nonattendance of ARVs at the prosperity workplaces was a critical demotivating factor, halting new ART clients. Many saw that some certified clients who were anxious to start ART on time on occasion expected to hold on for a significant long time in light of stock-outs of ARVs at the ART people group, making them feel cripple and rather relying upon close by flavors and traditional medicine. The people who are resolved to have HIV and are not yet qualified for ART due to great safe status are encouraged to go for routine seeing of their resistant status, for instance pre-ARV care, to start ART on time before getting interesting. Incident to follow-up during pre-ARV care was found to be huge for late ART beginning. Various ideal initiators had been under a type of pre-ARV care which enabled them to be consistently checked, and started on ART on time. Seen nonappearance of trust in some prosperity workers was consistently referred to by PLHIV as a critical obstacle to advantageous origin of ART. Some late initiators reported data on prosperity staff occasionally inspecting clients' HIV status out in the open, and communicated that this was the guideline reason behind them and various clients doing whatever it takes not to search for care at the HIV offices.

\section{Lack of Social Support System}

Social assistance from the nearby family or the neighborhood a standard was in like manner basic for advantageous or late ART beginning. Some late initiators completely missed the mark on a casual association that could maintain them financially or in their dynamic for searching for early thought. Females were on various occasions bound to begin ART very late appeared differently in relation to folks. More settled clients had a lower threat of beginning ART amazingly late diverged from energetic clients. Low taught clients were close to different occasions bound to begin ART late diverged from clients who were cultivated. Clients who were unmarried were on numerous occasions bound to begin ART late appeared differently in relation to the people who were hitched .Subsistence farmers were on numerous occasions bound to begin ART especially late stood out from non-farmers. Clients who required family maintain were on different occasions bound to begin ART late diverged from the people who had family maintain. Clients who had apparently not went to pre-ARV care were on various occasions bound to begin ART especially late appeared differently in relation to the people who had gone to pre-ARV care. Moreover, clients who had purportedly searched for care from ordinary/powerful healers preceding searching for formal ART care were on various occasions bound to begin

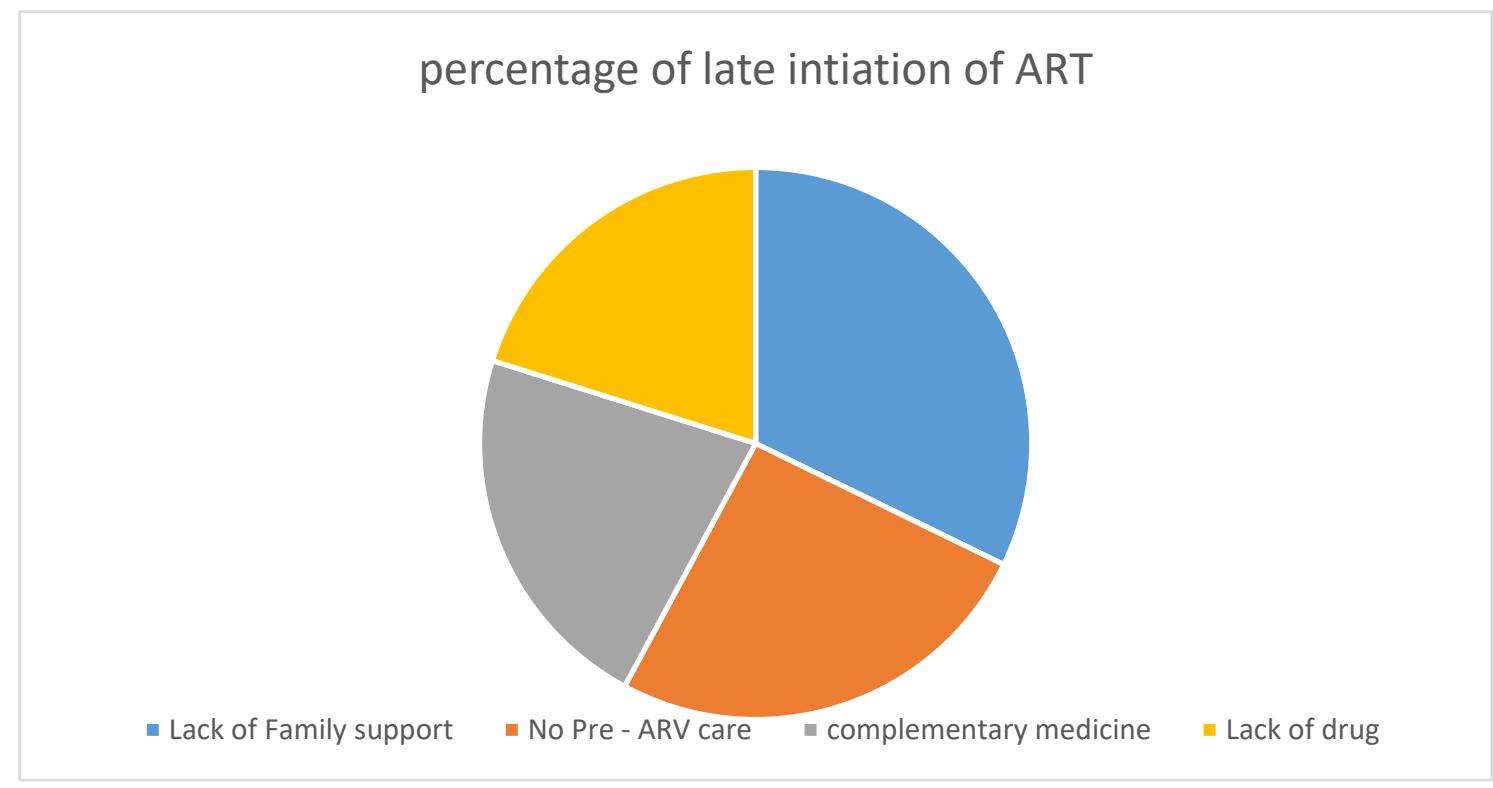

Fig.1:

ART particularly late appeared differently in relation to the people who didn't search for prior standard thought.

\section{Conclusion}

The difficulty to follow-up of HIV in transit from HIV exhorting and testing to pre-ARV care and resulting beginning of ART isn't just a part of the longing of the 
HIV, anyway an unpredictability of entwined factors going from an insufficient prosperity structure to particular/neighborhood decisions about HIV care. The essential prosperity system factors included consolidate inadequate post-test controlling, nonattendance of pre-ARV care for as of late screened HIV, competition from standard and extraordinary healers, ARV stock-outs and saw nonappearance of staff protection. The individual/neighborhood or misinterpretations that foil normal pre-ARV care or ideal origin of ART join disrespect and related nonattendance of family/social assistance, sexual direction unevenness (particularly impacting women) and confused decisions about ARVs. In the socio ecological model, the longing of the PLHIV to search for care falls under the individual level, the insufficient prosperity structure falls under the progressive and public methodology level, while neighborhood about HIV care fall under the social, definitive and neighborhood. The revelations as such address overhauling take-up of pre-ARV care, restricting adversity to follow-up of PLHIV and improving advantageous initiation of ART will require revolve around a couple of levels: the PLHIV, the deficient prosperity structure, the neighborhood other social common parts to make broad mediations that intentionally target instruments of needed change at every level of impact. Expanded prompting by staff with flitting planning in fundamental managing capacities got together with home visits by volunteer neighborhood maintain experts for advancing coordinating is connected with take-up of common pre-ARV care.

\section{References}

1. Chung MH, McGrath MJ, Beck IA, Levine M, Milne RS, So I, et al. Evaluation of the management of pretreatment HIV drug resistance by oligonucleotide ligation assay: a randomised controlled trial. Lancet HIV 2019. doi: 10.1016/S2352-3018 (19)30337-6.

2. Panpradist N, Beck IA, Vrana J, Higa N, Mclntyre D, Ruth PS, et al. OLA-simple: a software-guided HIV-1 drug resistance test for low-resource laboratories. EBioMedicine 2019. doi: 10.1016/j.ebiom.2019.11.002.

3. Cohen MS, Gay C, Kashuba AD, Blower S, Paxton L. Narrative review: antiretroviral therapy to prevent the sexual transmission of HIV-1. Ann Intern Med. 2007;146(8):591-601

4. Katz MH, Schwarcz SK, Kellogg TA, et al. Impact of highly active antiretroviral treatment on HIV seroincidence among men who have sex with men: San Francisco. Am J Public Health. 2002;92(3):388-394.
5. Bateganya M, Colfax G, Shafer LA, et al. Antiretroviral therapy and sexual behavior: a comparative study between antiretroviral-naive and -experienced patients at an urban HIV/AIDS care and research center in Kampala, Uganda. AIDS Patient Care STDS. 2005;19(11):760-768.

6. Stokols, D., Allen, J. \& Bellingham, R. L. 1996. The social ecology of health promotion: implications for research and practice. Am J Health Promot, 10, 24751.

7. Stolberg, H. O., Norman, G. \& Trop, I. 2004. Randomized controlled trials. Am J Roentgenol, 183, 1539-44.

8. Taegtmeyer, M. 2004. What is Voluntary Counselling and Testing (VCT)?, Nairobi, African Health Research HIV Forum, www.ncbi.nlm.nih.gov. Accessed 7th July 2009.

9. Nicholson, O., Mellins, C., Dolezal, C., Brackis-Cott, E. \& Abrams, E. J. 2006. HIV treatment-related knowledge and self-efficacy among caregivers of HIVinfected children. Patient Educ Couns, 61, 405-10. 